\title{
Los profesionales de la Psicopedagogía en la atención a la diversidad como Agente Educativo
}

\section{Psychopedagogy professionals in attention to diversity as an Educational Agent}

\author{
Graciela Zambrano Pincay \\ grace22zambrano@gmail.com \\ Universidad Técnica de Manabí \\ Ecuador \\ https://orcid.org/0000-0003-2396-3451 \\ Patricio Alfredo Vallejo Valdivieso \\ patricio 2871@yahoo.es \\ Universidad Técnica de Manabí \\ Ecuador \\ https://orcid.org/0000-0003-3248-7864 \\ Patricio Yosué Vallejo Pilligua \\ pvallejo@utm.edu.ec \\ Pontificia Universidad Católica del Ecuador \\ Ecuador \\ https://orcid.org/0000-0001-9039-7061 \\ Gelen Melissa Bravo Cedeño \\ helen.xiqi@hotmail.com \\ Universidad Técnica de Manabí \\ Ecuador \\ https://orcid.org/0000-0002-7401-7070
}

Recibido: 20 de abril del 2019

Aprobado: 31 de mayo del 2019

\section{RESUMEN}

El trabajo multidisciplinar en el proceso de atención a los niños y niñas con Necesidades Educativas Especiales (NEE) debe ser liderizado por el Psicopedagogo cuyas competencias permitirán garantizar un proceso de inclusión gradual en la sociedad. Este artículo de reflexión se plantea presentar el papel medular de apoyo y colaboración del Psicopedagogo, entre los agentes educativos para fomentar la inclusión integral de niños y niñas, e incluso a los diferentes niveles de edades, es decir a los adolescentes jóvenes, adultos y adultos mayores para su bienestar, 
desarrollo y aprendizaje. Se toma como referentes teóricos el aporte de autores en el área de la Psicopedagogía y desde una perspectiva introspectiva vivencial se fomenta el aporte del Psicopedagogo en la educación inclusiva.

Descriptores: Orientación pedagógica; Proceso de aprendizaje; Psicología del niño; Psicopedagogía; Atención.

\section{SUMMARY}

Multidisciplinary work in the process of attention to children with Special Educational Needs (EEN) must be led by the Psychopedagogue whose competences will ensure a gradual inclusion process in society. This reflection article proposes to present the core role of support and collaboration of the Psychopedagogue, among the educational agents to promote the integral inclusion of children, and even at different age levels, that is, to young adolescents, adults and adults. older for their well-being, development and learning. The contribution of authors in the area of Psychopedagogy is taken as theoretical references and, from an experiential introspective perspective, the contribution of Psychopedagogue in inclusive education is encouraged.

Descriptors: Educational guidance; Learning processes; Child psychology; Educational psychology; Attention .

\section{PROLEGÓMENO}

Para que exista el apoyo y colaboración, la educación debe ser no discriminatoria en términos de discapacidad, cultura y género; involucrando a todos los estudiantes en una comunidad sin excepciones; por tanto los estudiantes deben tener los mismos derechos para acceder al plan de estudios de valor cultural y todo el currículo inclusivo que ello conlleva.

El Centro Nacional de Reestructuración Educativa y la inclusión (1995), define la inclusión como:

La prestación de servicios a estudiantes con discapacidades, incluidos aquellos con discapacidades graves, en la escuela del vecindario, en clases de educación general apropiadas para su edad con el servicios de apoyo necesarios y ayudas complementarias para el niño y el maestro, tanto para asegurar el éxito del niño a nivel académico, de su comportamiento y aspectos sociales, para preparar al niño a que participe como miembro pleno y contribuyente de la sociedad. 
Se debe tener en cuenta que los bebés, niños y niñas menores de cinco años tienen contacto permanente con adultos que satisfacen sus necesidades básicas en la cotidianidad, entre ellos están en primer lugar sus padres, hermanos y otros miembros del núcleo familiar inmediato; pero, a la familia la acompañan otras personas en la crianza de sus hijos e hijas como son:

- El médico pediatra que revisa periódicamente su crecimiento, las enfermeras y otros prestadores de servicios de salud que le ponen las vacunas, etc.

- Los cuidadores, quienes de forma permanente o esporádica asumen la atención de los bebes, niños o niñas, ya sea en la casa del bebe o en la del cuidador .

- Personas que atienden a los niños y las niñas en espacios institucionales de primera infancia: educadores profesionales, auxiliares, personal de servicio, otros profesionales tales como psicólogos, etc. que dan apoyo especializado de acuerdo con las necesidades de los niños y las niñas.

- Vecinos, amigos de la familia y otros adultos que forman parte de la comunidad a la cual pertenece el niño o la niña y que constituyen su entorno.

Todas estas personas que interactúan de una u otra manera con el niño y la niña agencian su desarrollo, aunque no lo hagan intencionalmente, es por esto se les llama agentes educativos y están involucrados en la atención integral de niños y niñas menores de cinco años y es por estos que deben ejercer el principio de corresponsabilidad que nos impone la ley tanto a la familia, como al Estado y a la comunidad.

Dada la diversidad de perfiles que abarca el concepto de agente educativo (personal de servicio, miembros de la comunidad, familiares o profesionales que se encuentren vinculados al sector de protección, nutrición, salud o educación) es importante implementar acciones de formación que, con un enfoque intersectorial y diferenciado, hagan posible que cualquier interacción con los niños y las niñas tenga una intención educativa.

Se debe tener en cuenta, la importante tarea que desarrolla el personal que atiende a niñas y niños de 0 a 3 años de edad, en las diferentes instituciones de Educación 
Inicial , que podría delimitarse desde la profesionalización necesaria para el ejercicio de su tarea, hasta la definición de un perfil o el replanteamiento de su función en el contexto educativo actual, que son elementos significativos para ubicar las distintas dimensiones de su práctica; sin embargo, ser agente educativo está estrechamente ligado a una función sustancial, que en sí misma define y otorga características particulares a su labor cotidiana: la atención y educación de los niños más pequeños.

En los contextos familiar, institucional y/o comunitario, atender a bebés y niños pequeños implica reconocer cómo determinados procesos físicos, emocionales e intelectuales están entrelazados en su experiencia inmediata, en acciones que tal vez para la visión del adulto no resulten especialmente relevantes, porque no se perciben de manera directa, pero que para niñas y niños menores de tres años de edad, representan una oportunidad de aprendizaje, de conocer un mundo que a sus ojos resulta nuevo y difuso.

Un bebé, desde los primeros días de nacido, realiza un intenso proceso de exploración para organizar los sonidos, los lugares, los objetos, los colores, la presencia o ausencia de personas, los tiempos, las palabras, y todo lo que percibe a su alrededor; además, experimenta emociones y sentimientos que necesita descifrar y aprender a manejar.

En esta serie de descubrimientos el bebé requiere sentirse acompañado, sentir el contacto con personas que le proporcionan seguridad, le brindan oportunidades de comunicación, le miran con calidez y ponen a su alcance la posibilidad de conocerse, de sentirse seguro y capaz de ser parte de una comunidad y del espacio vital que le rodea, así, la tarea del agente educativo es propiciar ambientes estimulantes en donde los niños y las niñas se sientan incluidos en una red de relaciones, integrantes de un ambiente físico y emocionalmente seguro, donde el adulto esté dispuesto a favorecer un entorno de comunicación, investigación, participación, arte y juego que dé sustento al aprendizaje, al apoyarles con experiencias retadoras para que su cerebro organice progresivamente las estructuras que le permitan el desarrollo de sus capacidades. 
Por lo expresado, a los agentes educativos y a la inclusión debe verse como una interacción que se genera en el respeto hacia las diferencias individuales y las condiciones de participación desde una perspectiva de igualdad y equiparación de oportunidades sociales, cualesquiera que sean los valores culturales, la raza, el sexo, la edad y la condición de la persona o grupo de personas; es decir, es necesario, en una sociedad que lleve a cabo procesos de concienciación para comprender quiénes somos y con quiénes compartimos; se debe identificar y tratar a las personas tal cual son ellas mismas, una de ellas, el hijo de .., y además, asegurar que cada individuo comprenda que siempre hay alguien que los escucha y la entiende ; no necesariamente que le enseñe, pero sí que le comprenda.

En este sentido, es importante acotar que sin la inclusión recíproca básica de la persona con alguna condición discapacitante, y sus compañeros primarios, en espacios diádicos de unión afectivo social, no pueden surgir formas más avanzadas de unión humana (cognitivas, comunicativas y lingüísticas) en las que tengan lugar los procesos naturales de aprendizaje y desarrollo de las personas, de esta manera, los individuos con Necesidades Educativas Especiales (NEE) precisan ser incluidos de sus propios espacios: cognitivo, comunicativos y lingüísticos.

\section{DEL PROCESO DE INCLUSIÓN}

Todas las personas con NEE precisan tomar parte activa en situaciones, acontecimientos y experiencias que nutran la construcción de sus temas de aprendizaje y conversación.

Es importante prestar atención a los cambios que se deben hacer en el centro educativo, con el fin de adaptar el proceso de enseñanza a todo el alumnado, o de lo contrario, los esfuerzos puestos individualmente en la integración de los estudiantes, pueden resultar, a la postre, estériles. Al respecto, Miranda (2007) señala la "importancia a la participación en conjunto a los docentes, miembros de las instituciones educativas y sobre todo a la integración de los padres y madres de familia" (p. 25)

La Organización para la Cooperación y Desarrollo Económico (OCDE) en el 2019 sugiere a las instituciones educativas que se establezcan comunidades de 
aprendizaje, en donde todos los integrantes reflexionen y debatan sus conocimientos y experiencias, partiendo de que el conocimiento sobre el hecho educativo nunca está terminado, sino siempre es posible saber más, modificar o reforzar y, sobre todo, enriquecerse mutuamente.

A partir de lo expresado anteriormente, el rol de apoyo y colaboración del Psicopedagogo, entre los agentes educativos se han venido desarrollando satisfactoriamente en Manabí, ya que los centros de educación inicial están equipados y preparados física y metodológicamente, debido a que las Políticas de Estado que están brindando total apoyo a fomentar la inclusión integral de niños y niñas, e incluso a los diferentes niveles de edades, es decir a los adolescentes , jóvenes, adultos y adultos mayores; haciendo que el trabajo basado en la cooperación y la colaboración con el aprendizaje continuo para los agentes educativos, redunda en beneficio de los bebés y los niños, porque los objetivos son comunes y están enfocados a su bienestar, desarrollo y aprendizaje.

En una sociedad justa y equitativa no solo debe existir los centros escolares sencillos y simples como en la antigüedad, ya que el contexto moderno y humanitario solo comenzará con el reconocimiento de la naturaleza humana común a todas las personas, olvidando los estados de discriminación tales como los estereotipos, estigmatizaciones o poner etiquetas restrictivas, representación limitada o tergiversada, segregación, desigualdad de oportunidades y pérdida de derechos que hacían de la educación una camisa de fuerza para el desarrollo intelectual del ser humano, he allí, la importancia del currículo inclusivo y la inclusión integral y total de todas las personas en los diferentes campos biopsicosocial neuroendocrino .

La Educación Especial ha cambiado su panorama y por lo tanto, cambia también su forma de favorecer los procesos educativos de las personas con necesidades educativas especiales, pasando de desarrollar procesos asistenciales a procesos en los cuales se respeta la individualidad de las personas, en función de sus necesidades, características e intereses, y se pone énfasis en el entorno, como elemento que favorece o retrasa los procesos de participación de las personas con necesidades educativas especiales. 
La educación especial por lo tanto, debe reconceptualizarse a la luz de los procesos de integración, y no se debe concebir al estudiante con necesidades educativas especiales como aquel que tiene una característica individual o un déficit que le es propio , sino más bien, se debe tomar en cuenta la participación del entorno, las políticas gubernamentales, los aspectos sociales y educativos, que facilitan que las dificultades que la persona experimenta en su desarrollo socioeducativo y emocional continúen obstaculizando su desarrollo.

Señala Soto (2013) que Lou y López (2000) citando a Zabalza expresa que la integración escolar ha pasado por una serie de etapas, entre las cuales mencionan:

1. Reconocimiento del derecho a la educación de todos, sin embargo esto no reconoce que las personas con necesidades educativas especiales son "normales"

2. En relación con la anterior, la respuesta que se ha dado a estas personas es marginal y segregadora, por esta razón se han desarrollado servicios diferenciados en instituciones educativas,

\section{Aparece posteriormente la integración parcial}

El currículo de un entorno inclusivo es rico, interactivo, sensible a múltiples inteligencias y tiene muchos puntos de acceso. Un currículo bien diseñado se fundamenta en conocer a los alumnos destinatarios en toda su complejidad y en la certeza de que los contenidos impartidos son significativos y de interés cultural para todos.

La mayoría de las estrategias inclusivas tienen en común que son atractivas, interactivas, constructivistas que se sirven del conocimiento previo de los estudiantes y además fomentan y promueven el apoyo entre compañeros.

Para que la educación inclusiva sea una realidad, el profesorado debe adquirir la actitud, capacidad y conocimientos necesarios para enseñar a todos los estudiantes La inclusión no es simplemente una estructura organizativa, sino más bien el compromiso de hacer de las aulas, los centros escolares y el mundo lugares en los que todos los seres humanos sean valorados y bien acogidos, y donde la diversidad sea considerada enriquecedora y positiva. 


\section{EL ROL DEL PSICOPEDAGOGO}

Los y las psicopedagogos/as, en los establecimientos deben elaborar y tener programa de inclusión, como intención a la intervención a través de estrategias de aprendizaje, mediación para mediatizar los aprendizajes y hacer que trasciendan fuera del aula, entendiendo que el aprendizaje es el objeto de estudio primordial de la tarea del psicopedagogo.

Es así que el trabajo colaborativo, siendo una de las principales herramientas definidas para mejorar la calidad de los aprendizajes de todos los estudiantes, especialmente de los que presentan NEE; este se puede considerar una metodología de enseñanza y de realización de la actividad educativa basada en la creencia de que el aprendizaje y el desempeño laboral se incrementan cuando se desarrollan destrezas cooperativas para aprender y solucionar los problemas y acciones educativas y laborales en las cuales nos vemos inmersos.

La Psicopedagogía es una disciplina que se encarga de los fenómenos de orden psicológico para llegar a una formulación respecto a los métodos didácticos y pedagógicos; su enfoque se traduce en los fundamentos del sujeto y del objeto de conocimiento y su interrelación con el lenguaje, así como también la influencia socio cultural que tiene o recibe el individuo. Esta disciplina permite estudiar cómo el sujeto aprende respecto a sus procesos cognitivos y socio afectivos en las distintas etapas de su aprendizaje a lo largo de su vida, es por eso que a la psicopedagogía también se le define como: "Una especialización diferenciada e interdisciplinaria de la educación común, encaminada a orientar a los niños que en forma transitoria o permanente están impedidos de seguir el sistema de educación normal" (La importancia de la Psicopedagogía).

El Diccionario de la Real Academia Española define la psicopedagogía como Psicol. Rama de la psicología que se ocupa de los fenómenos de orden psicológico para llegar a una formulación más adecuada de los métodos didáctico y pedagógicos.", es por eso que Careaga (S/F) ha definido la psicopedagogía como "la disciplina que estudia la naturaleza y los procesos del aprendizaje humano formal y no formal, contextualizado y sus alteraciones". 
Se espera de cada psicopedagogo, que sea capaz de prevenir, evaluar e intervenir, de forma analítica y critica, a personas que presenten dificultades en sus aprendizajes; también debe estar capacitado para trabajar junto a equipos multidisciplinarios que centren su acción en el desarrollo de los procesos de enseñanza y aprendizaje tanto en la educación formal , así como también en la no formal.

En definitiva el profesional de la psicopedagogía interviene en el proceso de aprendizaje en personas que presentan dificultades, considerando no sólo su metodología de aprendizaje sino también desde un enfoque ecológico, el medio social y afectivo en que se desenvuelven.

Su objetivo es que logren aquellas habilidades que se encuentran deficientes; comprende el funcionamiento cerebral, desde el punto de vista de los procesos cognitivos y socio afectivos que intervienen en el aprendizaje; analizando las distintas perspectivas metodológicas referidas al aprendizaje de la lectura, escritura y cálculo, y conoce los procesos psicológicos y cognitivos que subyacen a estos aprendizajes; todo ello, en función del diseño e implementación de estrategias psicopedagógicas que permitan la intervención de las dificultades que pueden presentarse en estas áreas curriculares.

Los psicopedagogos desde su quehacer, no se podría relacionar con la didáctica de lo que enseña cualquier profesor, pero si intervienen desde los procesos de enseñanza aprendizaje, para lo cual hay estrategias de intervención propias del área, así que debemos ir más allá y abordarlo como mediador de aprendizajes con el conocimiento de las etapas del desarrollo biológico de cualquier estudiante, pero sin nunca perder de vista que ha de cumplir el rol de intermediario entre el conocimiento y el estudiante, siendo un puente construido con significancia del aprendizaje, intencionalidad y trascendencia, pues lo que se construye con el estudiante es para toda su vida y no queda aislado en áreas escolares, más bien se traspone a todas las áreas del conocimiento. 


\section{CONSIDERACIONES REFLEXIVAS}

El profesional en psicopedagogía, tiene que tener un bagaje de conocimientos intelectuales, académicos y científicos para poder orientar, ayudar y prevenir algunas alteraciones psicosociales que perjudiquen el desarrollo y crecimiento integral de las personas y más aun tratándose de menores, ya que ellos guardan y almacenan toda la información recibida para luego plasmarla, expresarlas y aflorarlas en su etapa adulta y profesional.

El psicopedagogo podrá elaborar un plan individualizado, tomando en cuentas las competencias que se tiene y lo que se quiere alcanzar, con lo que se logrará estructurar el pensamiento y los saberes.

Se puede encontrar trastornos del aprendizaje como la dislexia que es el deterioro de la capacidad para reconocer palabras, con lectura lenta e insegura y poca comprensión de lo leído; la disgrafía es el trastorno a la escritura, disortografía que es la presencia de grandes dificultades entre la asociación del código escrito, normas ortográficas con la escritura de las palabras; siendo la discalculia el trastorno al aprendizaje del conocimiento aritmético básico.

Una vez que el docente descubre alguna alteración en el aprendizaje, junto con el docente de apoyo y en especial el psicopedagogo tendrá que actuar en forma metodológica y científica para superar con estrategias y estilos metodológicos para corregir esas dificultades y si el caso lo amerita tendrá que solicitar apoyo multidisciplinario para llegar a cumplir lo planificado en su currículo inclusivo. 


\section{REFERENCIAS CONSULTADAS}

1. Ainscow, M., Booth, T., y Dyson, A. (2006). Mejorar las escuelas, desarrollar la inclusión. Londres: Routledge.

2. Liga Anti-Difamación.

Citado

como www.adl.org/education/courttv/pyramid_of_h ate.pdf

3. Armstrong, D., Armstrong, A. C., y Spandagou, I. (2011). Inclusión: ¿Por elección o por casualidad? Revista Internacional de Educación Inclusiva, 15, 29-39. doi: 10.1080 / 13603116.2010.496192

4. Ball, E. y Harry, B. (1993). Educación multicultural y educación especial: intersecciones y divergencias. Foro educativo, 57 (4), 430-437.

5. Ballard, K. (1997). Investigación sobre discapacidad y educación inclusiva: participación, construcción e interpretación. Revista Internacional de Educación Inclusiva, 1, 243-256. doi: 10.1080 / 1360311970010302

6. Junta de Educación-Distrito Escolar Unificado de la Ciudad de Sacramento v. Holanda. (1994). Obtenido de http://www.eric.ed.gov/PDFS/ED383135.pdf

7. Careaga, R. (s.f.). Hacia un concepto de Psicopedagogía. Recuperado de http://psicopedagogia.weebly.com/uploads/6/8/2/3/6823046/hacia un concept o de psicopedagogia.pdf

8. Centro Nacional de Reestructuración e Inclusión Educativa (NCERI). (1995). Estudio nacional de educación inclusiva. Nueva York: universidad de prensa de Nueva York.

9. Connor, D.J. y Ferri, B.A. (2007). El conflicto interno: resistencia a la inclusión y otras paradojas en la educación especial. Discapacidad y Sociedad, 22 (1), 63-77.

10. Danforth, S., y Rhodes, W. C. (1987). Deconstruyendo la discapacidad. Educación especial y de remediación, 18, 357-366.

11.Daniel R. R. v. Junta Estatal de Educación. (1989). Obtenido de http://www.maine.gov/educa tion / speced / cds / training / misceláneo / danielrr.pdf

12. Erevelles, N., Kanga, A. y Middleton, R. (2006). ¿Cómo se siente ser un problema? Raza, discapacidad y exclusión en la política educativa. En E. Brantlinger (Ed.). ¿Quién se beneficia de la educación especial? Remediación [arreglando] a los hijos de otras personas (pp. 77-99). Mahwah, NJ: Lawrence Erlbaum. 
13. Ferguson, D. L. (1995). El verdadero desafío de la inclusión: confesiones de un "inclusivo inclusivo". Phi Delta Kappan, 77, 281-287.

14. Ferri, B.A. (2010). Un diálogo que aún tenemos que tener: Estudios de raza y discapacidad. en C. DudleyMarling y A. Gurn. El mito de la curva normal (Ch.10). Nueva York: Peter Lang.

15. Ferri, B.A. y Connor, D.J. (2005). Herramientas de exclusión: raza, discapacidad y educación (re) segregada. Registro del Colegio de Maestros. 107 (3), 453-474.

16. Ferri, B.A. y Connor, D.J. (2006). Resistencia a la lectura: discursos de exclusión en los debates de desagregación e inclusión. Nueva York: Peter Lang.

17. Gabel, S. L., y Danforth, S. (2008). La discapacidad y la política internacional de la educación. En S. L. Gabel y S. Danforth (Eds.), La discapacidad y la política de la educación: un lector internacional (pp. 1-18). Nueva York, NY: Peter Lang.

18. Idol, L. (2006). Hacia la inclusión de los estudiantes de educación especial en la educación general: un programa de evaluación de ocho escuelas. Remedios y educación especial, 27, 77-94. doi: 10.1177 / 07419325060270020601

19. Junta de Educación-Distrito Escolar Unificado de la Ciudad de Sacramento v. Holanda. (1994). Obtenido de http://www.eric.ed.gov/PDFS/ED383135.pdf

20. La importancia de la Psicopedagogía. (s.f.). Recuperado de http:// https://psicopedagogasdelfuturo.wordpress.com/blog/

21. Lawrence-Brown, D. y Sapon-Shevin, M. (2013). Condición crítica: Principios clave para una educación equitativa e inclusiva. NY: Teachers College Press.

22. Ley de Educación para Personas con Discapacidad. 20 U.S.C. § 1412. (2004). Recuperado de http: // idea. ed.gov/explore/view/p/,root,regs,300,B,300\%252E114

23. Liga anti-difamación. Citado como www.adl.org/education/courttv/pyramid of $h$ ate.pdf

24. MIRANDA-CARVAJAL, Carlos Ernesto. Local cultural heritage as a tool for education. Rev. Int. Investig. Cienc. Soc. [online]. 2017, vol.13, n.1, pp.25-34. ISSN 2226-4000. http://dx.doi.org/10.18004/riics.2017.julio.25-34. 
25. Organización de las Naciones Unidas para la Educación, la Ciencia y la Cultura (UNESCO). (1994, 7-10 de junio). La Declaración de la UNESCO de Salamanca. Adoptado por la Conferencia Mundial sobre educación especial: acceso y calidad, Salamanca, España. Obtenido de http://www.csie.org.uk/inclu sion / unesco-salamanca.shtml

26. Organización de las Naciones Unidas para la Educación, la Ciencia y la Cultura (UNESCO). (2000). Foro mundial de la educación: el marco de acción de Dakar. Obtenido de http://unesdoc.unesco.org/ images / 0012/001211 / 121147e.pdf

27. Organización para la Cooperación y Desarrollo Económico (OCDE). (2019). Innovation in education: Midiendo la innovación en la educación 2019, ¿Qué ha cambiado en la sala de clases? Recuperado de http: https://chile.gob.cl/ocde/informes-ocde/otras-publicaciones-ocde/innovation-ineducation-midiendo-la-innovacion-en-la-educacion-2019+

28. Pugach, M. y Seidl, B. (1998). Vínculos responsables entre diversidad y discapacidad: un desafío para la educación especial. Educación Docente y Educación Especial, 21 (4), 319-333.

29. Pugach, M.C., Blanton, L.P. y Florian, L. (2012). Conversaciones inquietantes: diversidad y discapacidad en la formación del profesorado. Diario de la formación del profesorado, 63 (4), 235-236.

30. Sailor, W., Gerry, M., y Wilson, W. C. (1990). Implicaciones políticas de los modelos emergentes de inclusión total para la educación de estudiantes con discapacidades graves (Informe No. EC302673). San Francisco, CA: Universidad Estatal de San Francisco. Servicio de reproducción de documentos Eric No. ED 365048.

31.Sapon-Shevin, M. (1996). Inclusión completa como tableta reveladora: Revelando las fallas en nuestro sistema de educación general. Teoría enPráctica, 35 (1), 35-41.

32. Sapon-Shevin, M. (2007). Ampliando el círculo: El poder de las aulas inclusivas. Boston: Beacon Press.

33. Sapon-Shevin, M. (2010). Porque podemos cambiar el mundo: una guía práctica para crear comunidades de aulas cooperativas e inclusivas. Thousand Oaks, CA: Corwin Press.

34. Shyman, E. (2013). Más allá de la igualdad en el aula estadounidense: el caso de la educación inclusiva. Lanham, MD: Lexington Books. 
35. Soto Calderón, Ronald, La inclusión educativa: Una tarea que le compete a toda una sociedad . Revista Electrónica "Actualidades Investigativas en Educación" [en linea] 2003, 3 (enero-junio): [Fecha de consulta: 23 de julio de 2019] Disponible en: $\leq$ http://www.redalyc.org/articulo.oa?id=44730104> ISSN

36. Ware, L. P. (2002). Una conversación moral sobre la discapacidad: arriesgando lo personal en contextos educativos. Hipatia, 17, 143-172. doi: $10.1111 /$ j.1527-2001.2002.tb00945.x

\section{REFERENCES CONSULTED}

1. Ainscow, M., Booth, T., and Dyson, A. (2006). Improve schools, develop inclusion. London: Routledge.

2. Anti-Defamation

League.

Cited

as www.adl.org/education/courttv/pyramid_of_h ate.pdf

3. Armstrong, D., Armstrong, A. C., and Spandagou, I. (2011). Inclusion: By choice or by chance? International Journal of Inclusive Education, 15, 29-39. doi: 10.1080 / 13603116.2010 .496192

4. Ball, E. and Harry, B. (1993). Multicultural education and special education: intersections and divergences. Educational forum, 57 (4), 430-437.

5. Ballard, K. (1997). Research on disability and inclusive education: participation, construction and interpretation. International Journal of Inclusive Education, 1, 243-256. doi: 10.1080 / 1360311970010302

6. Board of Education - Sacramento City Unified School District v. Holland. (1994). Retrieved from http://www.eric.ed.gov/PDFS/ED383135.pdf

7. Careaga, R. (s.f.). Towards a concept of Psychopedagogy. Retrieved from http://psicopedagogia.weebly.com/uploads/6/8/2/3/6823046/hacia_un_concept o_de_psicopedagogia.pdf

8. National Center for Educational Restructuring and Inclusion (NCERI). (nineteen ninety five). National study of inclusive education. New York: New York press university.

9. Connor, D.J. and Ferri, B.A. (2007). The internal conflict: resistance to inclusion and other paradoxes in special education. Disability and Society, 22 (1), 63-77.

10. Danforth, S., and Rhodes, W. C. (1987). Deconstructing disability. Special and remediation education, $18,357-366$. 
11.Daniel R. R. V. State Board of Education. (1989). Obtained from http://www.maine.gov/educa tion / speced / cds / training / miscellaneous / danielrr.pdf

12. Erevelles, N., Kanga, A. and Middleton, R. (2006). How does it feel to be a problem? Race, disability and exclusion in education policy. In E. Brantlinger (Ed.). Who benefits from special education? Remediation [fixing] the children of other people (pp. 77-99). Mahwah, NJ: Lawrence Erlbaum.

13. Ferguson, D. L. (1995). The real challenge of inclusion: confessions of an "inclusive inclusive". Phi Delta Kappan, 77, 281-287.

14. Ferri, B.A. (2010). A dialogue that we still have to have: Race and disability studies. in C. DudleyMarling and A. Gurn. The myth of the normal curve (Ch.10). New York: Peter Lang.

15. Ferri, B.A. and Connor, D.J. (2005). Exclusion tools: race, disability and (re) segregated education. Registration of the Teachers College. 107 (3), 453-474.

16. Ferri, B.A. and Connor, D.J. (2006). Reading resistance: exclusion speeches in disaggregation and inclusion debates. New York: Peter Lang.

17. Gabel, S. L., and Danforth, S. (2008). Disability and international education policy. In S. L. Gabel and S. Danforth (Eds.), Disability and education policy: an international reader (pp. 1-18). New York, NY: Peter Lang.

18. Idol, L. (2006). Towards the inclusion of special education students in general education: an evaluation program of eight schools. Remedies and special education, 27, 77-94. doi: 10.1177 / 07419325060270020601

19. Board of Education - Sacramento City Unified School District v. Holland. (1994). Retrieved from http://www.eric.ed.gov/PDFS/ED383135.pdf

20. The importance of Psychopedagogy. (s.f.). Retrieved from http: // https://psicopedagogasdelfuturo.wordpress.com/blog/

21. Lawrence-Brown, D. and Sapon-Shevin, M. (2013). Critical condition: Key principles for equitable and inclusive education. NY: Teachers College Press.

22. Education Law for People with Disabilities. 20 U.S.C. § 1412. (2004). Recovered from http: I/ idea. ed.gov/explore/view/p/,root,regs,300,B,300\%252E114

23. Anti-Defamation League Cited as www.adl.org/education/courttv/pyramid_of_h ate.pdf 
24. MIRANDA-CARVAJAL, Carlos Ernesto. Local cultural heritage as a tool for education. Rev. Int. Investig. Science. Soc. [Online]. 2017, vol.13, n.1, pp. 2534. ISSN 2226-4000. http://dx.doi.org/10.18004/riics.2017.July 25-34.

25. United Nations Educational, Scientific and Cultural Organization (UNESCO). (1994, June 7-10). The UNESCO Declaration of Salamanca. Adopted by the World Conference on special education: access and quality, Salamanca, Spain. Obtained from http://www.csie.org.uk/inclu sion / unescosalamanca.shtml

26. United Nations Educational, Scientific and Cultural Organization (UNESCO). (2000). World education forum: the Dakar framework for action. Retrieved from http://unesdoc.unesco.org/ images / 0012/001211 / 121147e.pdf

27. Organization for Economic Cooperation and Development (OECD). (2019). Innovation in education: Measuring innovation in education 2019, what has changed in the classroom? Retrieved from http: https://chile.gob.cl/ocde/informes-ocde/otras-publicaciones-ocde/innovation-ineducation-midiendo-la-innovacion-en-la-educacion-2019+

28. Pugach, M. and Seidl, B. (1998). Responsible links between diversity and disability: a challenge for special education. Teacher Education and Special Education, 21 (4), 319-333.

29. Pugach, M.C., Blanton, L.P. and Florian, L. (2012). Disturbing conversations: diversity and disability in teacher training. Journal of teacher training, 63 (4), 235-236.

30.Sailor, W., Gerry, M., and Wilson, W. C. (1990). Political implications of emerging models of total inclusion for the education of students with severe disabilities (Report No. EC302673). San Francisco, CA: San Francisco State University. Document reproduction service Eric No. ED 365048.

31. Sapon-Shevin, M. (1996). Full inclusion as a revealing tablet: Revealing the failures in our general education system. Theory in Practice, 35 (1), 35-41.

32. Sapon-Shevin, M. (2007). Expanding the circle: The power of inclusive classrooms. Boston: Beacon Press.

33. Sapon-Shevin, M. (2010). Because we can change the world: a practical guide to create communities of cooperative and inclusive classrooms. Thousand Oaks, CA: Corwin Press.

34. Shyman, E. (2013). Beyond equality in the American classroom: the case of inclusive education. Lanham, MD: Lexington Books. 
35. Soto Calderón, Ronald, Educational inclusion: A task that falls to a whole society. Electronic Magazine "Investigative News in Education" [online] 2003, 3 (January-June): [Date of consultation: July 23, 2019] Available at: <http://www.redalyc.org/articulo.oa? id $=44730104>$ ISSN

36. Ware, L. P. (2002). A moral conversation about disability: risking the personal in educational contexts. Hypatia, 17, 143-172. doi: 10.1111 / j.15272001.2002.tb00945.x

2019 por los autores. Este artículo es de acceso abierto y distribuido según los términos y condiciones de la licencia Creative Commons Atribución-NoComercial-Compartirlgual 4.0 Internacional (CC BY-NC-SA 4.0) (https://creativecommons.org/licenses/by-nc-sa/4.0/). 